\title{
On impulse excitation of the global poloidal modes in the magnetosphere
}

\author{
P. N. Mager and D. Yu. Klimushkin \\ Institute of Solar-Terrestrial Physics (ISTP), Russian Academy of Science, Siberian Branch, Irkutsk, 664033, Russia \\ Received: 24 July 2006 - Revised: 29 August 2006 - Accepted: 31 August 2006 - Published: 20 October 2006
}

\begin{abstract}
Through the combined action of the field line curvature and finite plasma pressure in some regions of the magnetosphere (plasmapause, ring current) there can exist global poloidal Alfvén modes standing both along field lines and across magnetic shells and propagating along azimuth. In this paper we investigate the spatio-temporal structure of such waves generated by an impulsive source. In general, the mode is the sum of radial harmonics whose structure is described by Hermitian polynomials. For the usually observed second harmonic structure along the background field, frequencies of these radial harmonics are very close to each other; therefore, the generated wave is almost a monochromatic oscillation. But mixing of the harmonics with different radial structure causes the evolution of the initially poloidal wave into the toroidal one. This casts some doubts upon the interpretation of observed high- $m$ poloidal waves as global poloidal modes.
\end{abstract}

Keywords. Magnetospheric physics (MHD waves and instabilities; Plasmasphere) - Space plasma physics (Kinetic and MHD theory)

\section{Introduction}

The wide variety of Alfvén waves in the Earth's magnetosphere includes frequently recorded poloidally polarized oscillations (the magnetic field vector oscillates radially). These waves have large values of the azimuthal wave number $m \gg 1$, and they are usually second longitudinal harmonic standing waves (that is, a second harmonic structure along the background field), being often narrowly localized across magnetic shells (Cramm et al., 2000; Takahashi and Anderson, 1992; Yeoman et al., 2000). These oscillations are often interpreted in terms of global poloidal modes (Leonovich and

Correspondence to: P. N. Mager

(p.mager@iszf.irk.ru)
Mazur, 1995; Vetoulis and Chen, 1996; Denton and Vetoulis, 1998; Klimushkin, 1998; Mager and Klimushkin, 2002; Denton et al., 2003; Klimushkin at al., 2004). These modes represent waveguides, where the wave energy is trapped between the conjugate ionospheres and the cut off shells, and is channelled along azimuth. They can exist near the plasmapause and in ring current regions inside and outside of the plasmasphere, where the poloidal frequency $\Omega_{P N}$ as a function of coordinate across magnetic shells $\left(x^{1}\right)$ has extreme values (Fig. 1). The global poloidal modes have discrete spectrum (even in the ideal MHD), due to the field line curvature and finite plasma pressure.

It is widely accepted that poloidal waves have an energy source in energetic particles (e.g. Ozeke and Mann, 2001). However, corresponding kinetic instabilities are the amplification mechanism of the waves rather than their source. In order for this mechanism to work, it is necessary to have some "embryos" of the oscillations. Since the ULF waves are not permanent features of the magnetosphere, this triggering amplitude must be a transient process. Thus, the study of nonstationary Alfvén waves is of interest. A number of publications (e.g. Tataronis and Grossman, 1973; Mann and Wright, 1995; Leonovich and Mazur, 1998) have addressed the problem of the evolution of the Alfvén wave field from an initial poloidally polarized packet in magnetospheric regions with a monotonic dependence of magnetospheric parameters on the transverse coordinate. It was shown, in particular, that although at the initial instant of time all field lines oscillate with the same phase, the oscillations on neighboring magnetic shells rapidly acquire a significant phase difference, because each field line oscillates with its own eigenfrequency (phase mixing phenomenon). As a consequence, the wave becomes strongly "indented" in radial coordinate and, hence, toroidally polarized. The reason for the phase mixing is the continuous spectrum Alfvén waves: for every given frequency $\omega$ we can find an L-shell with the Alfvén eigenfrequency being equal to $\omega$.

Published by Copernicus GmbH on behalf of the European Geosciences Union. 

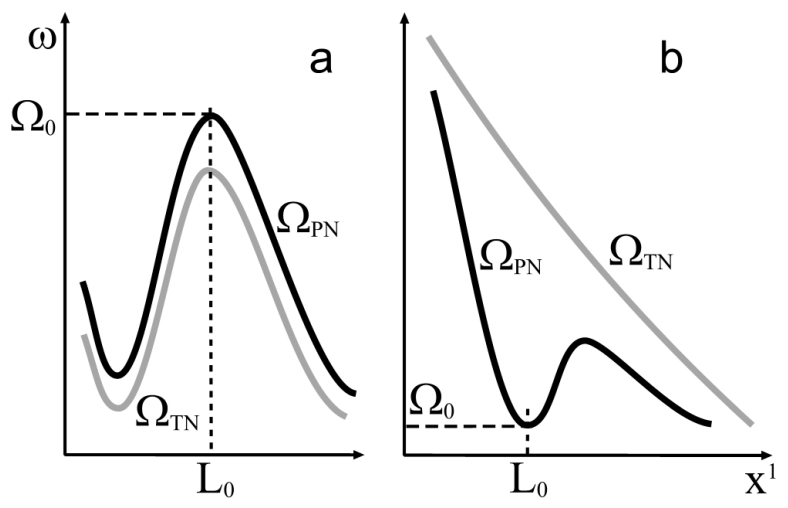

Fig. 1. Dependence of the toroidal $\Omega_{T N}$ and poloidal $\Omega_{P N}$ frequencies on the transverse coordinate $x^{1}$ in the region of the waveguide on the plasmapause (a) and near the ring current (b).

The prime objective of the present paper is the study of the temporal evolution of the global poloidal modes with a discrete spectrum. We are going to elucidate on whether the impulse-excited global poloidal modes remain poloidal forever, or whether they are also transformed into toroidally polarized waves. In the latter case, some doubts are cast upon the interpretation of the observed poloidal waves as global poloidal modes.

We study the impulsive excitation of poloidally polarized global Alfvén poloidal modes by a source whose operation time is much less than the characteristic period of these oscillations and whose characteristics change little across magnetic shells in the region of their localization. Our analysis is based on the theory of Alfvén eigenoscillations of the axisymmetric magnetosphere in which the field line curvature, the plasma nonuniformity along and across the magnetic field, and finite plasma pressure are taken into account (Leonovich and Mazur, 1995; Klimushkin at al., 2004).

\section{Transverse structure}

We now summarize the main results from the theory of monochromatic poloidal standing oscillations across magnetic shells in the axisymmetric magnetosphere (Leonovich and Mazur, 1995; Klimushkin et al., 2004). Let us designate the radial coordinate $x^{1}$, then $\xi$ is its dimensionless form (as specified below). As is shown in the cited references, the equation describing the transverse structure of Alfvén waves in the waveguide is of the form

$$
\frac{d^{2} R_{N}}{d \xi^{2}}+\left(\sigma-\xi^{2}\right) R_{N}=q_{N}
$$

Here $R_{N}\left(x^{1}\right)$ describes the radial structure of the mode, where it is proportional to the wave electric field azimuthal

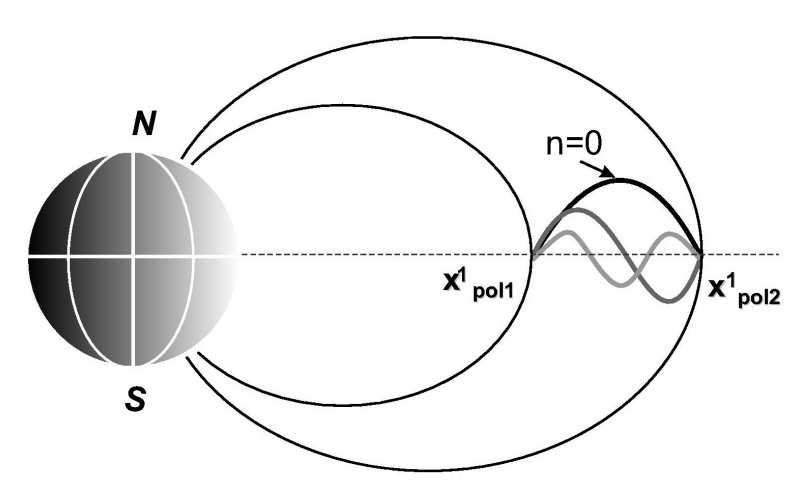

Fig. 2. Structure of the wave across magnetic shells in the Alfvén waveguide.

component; $N$ is the longitudinal harmonic number (usually observed $N=2$ ). We also have introduced the designations:

$\sigma=\frac{l^{2}}{\lambda_{R N}^{2}}\left(\frac{\omega^{2}-\Omega_{0}^{2}}{\Omega_{0}^{2}}\right)$;

$\lambda_{R N}=\left(\frac{l L_{0}}{m}\right)^{1 / 2}\left(\frac{\Omega_{0}^{2}-\Omega_{T N}^{2}}{\Omega_{0}^{2}}\right)^{1 / 4} ;$

$\xi=\left(x^{1}-L_{0}\right) / \lambda_{R N} ; N$ is the longitudinal harmonic number; $q_{N}$ is the function that specifies the oscillation source; $\Omega_{0}$ and $L_{0}$ are the values of the extremum of the function $\Omega_{P N}\left(x^{1}\right)$ and its position, respectively:

$\Omega_{P N}^{2}\left(x^{1}\right)=\Omega_{0}^{2}\left[1 \mp\left(\frac{x^{1}-L_{0}}{l}\right)^{2}\right] ;$

$\Omega_{T N}$ is the toroidal eigenfrequency that bounds the value of the wave frequency in the waveguide (see Fig. 1); and $\omega$ is the wave frequency.

If $q_{N}=0$, this equation is of the same form as is the Schrödinger equation for a harmonic oscillator. As is well known, there exists its solution bounded outside of the region of mode localization. This requires that the parameter $\sigma$ be quantized, $\sigma=2 n+1$, where $n=0,1,2, \ldots$ is an integer. From this we have the wave frequency quantization condition:

$\omega=\omega_{N n}, \omega_{N n}^{2} \equiv \Omega_{0}^{2} \mp \Omega_{0}^{2} \frac{\lambda_{R N}^{2}}{l^{2}}(2 n+1)$.

Here the "-" sign refers to the case where the waveguide is localized near the maximum of the function $\Omega_{P N}\left(x^{1}\right)$, and the " + " sign refers to opposite case. Thus, the oscillation has a discrete spectrum, as pointed out above. The difference between the eigenfrequencies $\omega_{N n}$ depends on the difference between poloidal and toroidal frequencies $\Delta \Omega=\Omega_{P N}-\Omega_{T N}$ in the waveguide region: the higher $\Delta \Omega$, the higher the difference between the eigenfrequencies. For the fundamental longitudinal harmonic $(N=1)$ this difference can be rather 


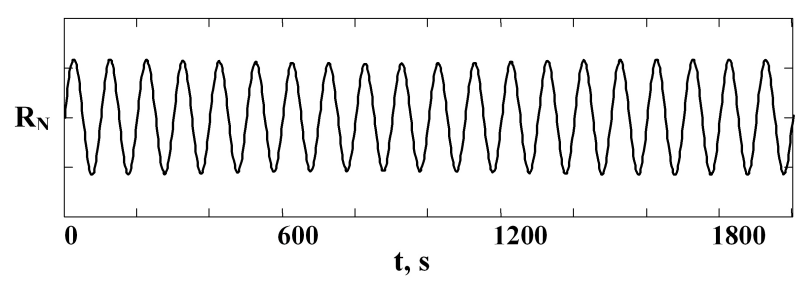

Fig. 3. Time-dependence of the wave field in the waveguide. Here $t$ is time after the impulse.

large, while for the more often observed second harmonic $(N=2)$ it is very small, $\Delta \Omega \ll \Omega_{P N}, \Omega_{T N}$ (Klimushkin et al., 2004), so $\lambda_{R 2}^{2} / l^{2} \ll 1$. The spectrum must be very dense in this case.

When $q_{N}=0$, the solution to Eq. (1) is expressed in terms of Hermitian polynomials $H_{n}$ :

$R_{N n}=$ const $\cdot \pi^{-1 / 4} 2^{-\mathrm{n} / 2}(\mathrm{n} !)^{-1 / 2} \mathrm{H}_{\mathrm{n}}(\xi) \mathrm{e}^{-\xi^{2} / 2}$.

This solution describes the transverse structure of the $n$-th harmonic of the waveguide (Fig. 2). In particular, the fundamental radial harmonic $(n=0)$ is described by the Gaussian function. If the source function $q_{N} \neq 0$, then the structure of the wave is defined by the expression

$R_{N}=\sum_{n=0}^{\infty} \frac{c_{n}}{\omega^{2}-\omega_{N n}^{2}} R_{N n}(\xi)$

where

$c_{n}=\int_{-\infty}^{\infty} R_{N n}(\xi) q_{N} d \xi$

(Leonovich and Mazur, 1995). Thus, with the nonzero righthand side, the equation of oscillations has the solution for any frequency, although the amplitude of the wave field is still maximal when $\omega \simeq \omega_{n}$.

Next, based on the solution Eq. (4), we shall study the evolution of the Alfvén wave excited by the impulsive source.

\section{Excitation of Alfvén oscillations in the waveguide by the impulsive source}

In order to determine how the wave field will change with the time, we Fourier-transform the solution Eq. (4):

$R_{N}\left(x^{1}, t\right)=\frac{1}{\sqrt{2 \pi}} \int_{-\infty}^{\infty} R_{N}\left(x^{1}, \omega\right) e^{-i \omega t} d \omega$.

It is assumed that the source function $q_{N}$ is independent of $x^{1}$. This approximation corresponds to the fact that the width of the waveguide is much less than the characteristic scale of the variation of the source characteristics across magnetic

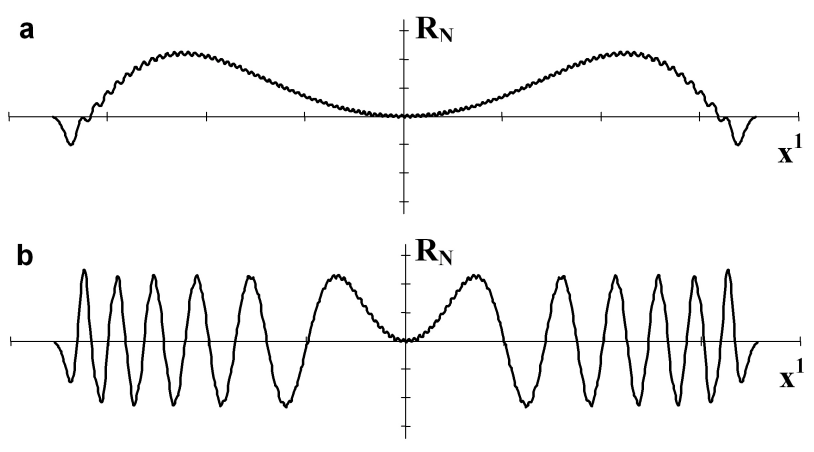

Fig. 4. Structure of the wave field across magnetic shells: (a) for $t=1000 \mathrm{~s}$ (10 periods approximately) after the impulse, (b) for $t=10000 \mathrm{~s}$ ( $\sim 100$ periods $)$ after the impulse.

shells. It is further assumed that $q_{N}$ is independent of $\omega$, which approximately corresponds to the fact that the operation time of the source is much less than the characteristic period of the oscillations under investigation, i.e. the source is an impulsive one. In the case under study, when $q_{N}\left(x^{1}, \omega\right)=$ const, the source is described by a $\delta$-function of time:

$\frac{1}{\sqrt{2 \pi}} \int_{-\infty}^{\infty} q_{N} e^{-i \omega t} d \omega=\sqrt{2 \pi} q_{N} \delta(t)$.

As a result, from Eq. (6) we find that

$R_{N}\left(x^{1}, t\right)=2 \sum_{j=0}^{\infty} \frac{c_{j}}{\omega_{N 2 j}} \sin \left(\omega_{N 2 j} t\right) R_{N 2 j}\left(x^{1}\right)$,

where

$c_{j}=q_{N} \pi^{-1 / 4} 2^{-j} \frac{\sqrt{(2 j) !}}{j !} \sqrt{2 \pi}$.

Here we have substituted the summation index $n$ for $j$ $(j=0,1, \ldots)$, because the terms of the series with $n=2 j+1$ are zero, i.e. only even $n$-harmonics of the waveguide are excited.

For the fundamental longitudinal harmonic $(N=1)$, the difference between the waveguide eigenfrequencies $\omega_{N n}$ is rather large, since in this case $\lambda_{R 1}^{2} / l^{2} \sim 1$. Thus, as can be seen from the Eq. (7), the amplitude of the radial harmonic with $n=0$ is considerably larger than the amplitudes of the higher radial harmonics. Consequently, the excited wave will have the frequency $\omega \approx \omega_{N 0}$ and its radial structure will be close to the Gaussian function. On the contrary, for the most often observed second longitudinal harmonic $(N=2)$, the difference between radial eigenfrequencies $\omega_{N n}$ is very small, since $\lambda_{R 2}^{2} / l^{2} \ll 1$ in this case, that also leads to the small difference of the different terms in Eq. (7).

In order to study the temporal and spatial evolution of the wave, we performed a numerical summation of the series Eq. (7) with the parameters $\lambda_{R 2}^{2} / l^{2}=3 \cdot 10^{-4}$, 
$\Omega_{0}=6.28 \cdot 10^{-2} s^{-1}$; the waveguide near the minimum of the poloidal frequency was considered (see Fig. 1b), so the sign "+" was chosen in Eqs. $(2,3)$. The numerical summation shows that the excited wave is almost monochoromatic (Fig.3), as in the case $N=1$, but the radial structure is timedependent: the radial wavelength is decreasing with time (Fig. 4). The reason for this analogy of the phase mixing is the great impact on the wave structure of the higher radial harmonics $(n>1)$. The ratio of the radial component $B_{1}$ of the Alfvén wave magnetic field to the azimuthal component $B_{2}$ is defined as

$$
\frac{B_{1}}{B_{2}} \approx \frac{m L^{-1} R_{N}}{\partial R_{N} / \partial x^{1}}
$$

The wave is poloidally polarized if $B_{1} / B_{2} \gg 1$, and it is toroidally polarized if $B_{1} / B_{2} \ll 1$. Accordingly, the wave polarization changes from poloidal to toroidal.

\section{Summary}

Let us describe the main results of this study. Within the Alfvén waveguide, the impulsive source excites virtually all waveguide radial harmonics. The distance between their eigenfrequencies strongly depends on the difference between poloidal and toroidal frequencies near the center of the waveguide. This difference is generally large for the fundamental harmonic structure along the background field $(N=1)$ and small for the second harmonic $(N=2)$. Accordingly, in the former case the relative difference between the frequencies of the nearest radial harmonics $\Delta \omega_{n}$ is rather large, $\Delta \omega_{n} \sim \omega$, and in the latter case the harmonics are very close to each other, $\Delta \omega_{n} \ll \omega$. In its turn, it determines the radial structure of the excited modes. In the $N=1$ case, the fundamental radial harmonic $(n=0)$ is predominantly excited, hence the radial structure of the oscillation almost coincides with its eigenfunction, the Gaussian function. The oscillation is quasi-monochromatic throughout entire lifetime of the wave, so the wave is poloidally polarized. For the second longitudinal harmonic $N=2$, all radial harmonics make almost an equal contribution to the wave structure. Soon after the impulse, the higher radial harmonics come into play and make the radial wavelength decrease with time. This leads to a transformation of the wave from poloidal to toroidal. In the $N=2$ case, the excited oscillation is still quasi-monochromatic, since the frequencies of the different harmonics are very close to each other.

It should be noted that observed poloidal Alfvén waves in the magnetosphere usually have the second rather than fundamental longitudinal harmonics (e.g. Takahashi and Anderson, 1992). The quick transformation of these modes to toroidal found in this paper makes it rather improbable their interpretation as waveguide modes, or global poloidal modes.

It must be mentioned that to our best knowledge there were no published observations of poloidal waves turning into toroidal waves; besides, no observed high- $m$ waves had toroidal polarization. The simplest possible explanation of this controversy is that poloidal waves are damped due to finite ionospheric conductivity, not having enough time for the transition.

If, as is usually supposed, high- $m$ waves are excited by various kinetic instabilities, the instability growth rate is the largest for the poloidally polarized waves (Klimushkin, 2000). But poloidal waves inevitable transform into toroidal ones. Although the rate of the wave enhancements is decreasing in the course of transformation, it still remains positive, so the most enhanced oscillations must be toroidally polarized. Because no high- $m$ toroidal pulsations have been observed, it casts some suspicion on the possibility of the instabilities to generate high- $m$ waves (see also Klimushkin and Mager, 2004; Mager and Klimushkin, 2005).

Acknowledgements. The work is partially supported by INTAS grant 05-1000008-7978, Russian Foundation for Basic Research grant 04-05-64321 and Program of presidium of Russian Academy of Sciences \#16 and OFN RAS \#16. The work of P. Mager was supported by Russian Science Support Foundation.

Topical Editor I. A. Daglis thanks T. Yeoman for his help in evaluating this paper.

\section{References}

Cramm, R., Glassmeier, K.-H., Othmer, C., Fornacon, K. H., Auster, H. U., Baumjohan, W., and Georgescu, E.: A case study of a radially polarized Pc 4 event observed by the Equator-S satellite, Ann. Geophys., 18, 411-415, 2000, http://www.ann-geophys.net/18/411/2000/.

Denton, R. E. and Vetoulis, G.: Global poloidal mode, J. Geophys. Res., 103, 6729-6739, 1998.

Denton, R. E., Lessard, M. R., and Kistler, L. M.: Radial localization of magnetospheric guided poloidal Pc 4-5 waves, J. Geophys. Res. 108(A3), 1105, doi:10.1029/2002JA009679, 2003.

Klimushkin, D. Yu.: Resonators for hydromagnetic waves in the magnetosphere, J. Geophys. Res., 103, 2369-2378, 1998.

Klimushkin, D. Yu.: The propagation of high- $m$ Alfvén waves in the Earths magnetosphere and their interaction with high-energy particles, J. Geophys. Res., 105, 23 303-23 310, 2000.

Klimushkin, D. Yu. and Mager, P. N.: The spatio-temporal structure of impulse-generated azimuthal small-scale Alfven waves interacting with high-energy charged particles in the magnetosphere, Ann. Geophys., 22, 1053-1060, 2004, http://www.ann-geophys.net/22/1053/2004/.

Klimushkin, D. Yu., Mager, P. N., and Glassmeier, K.-H.: Toroidal and poloidal Alfvén waves with arbitrary azimuthal wave numbers in a finite pressure plasma in the Earth's magnetosphere, Ann. Geophys., 22, 267-288, 2004, http://www.ann-geophys.net/22/267/2004/.

Leonovich, A. S. and Mazur, V. A.: Magnetospheric resonator for transverse-small-scale standing Alfvén waves, Planet. Space Sci., 43, 881-883, 1995.

Leonovich A. S. and Mazur, V. A.: Standing Alfvén waves in an axisymmetric magnetosphere excited by a non-stationary source, 
Ann. Geophys., 16, 914-920, 1998,

http://www.ann-geophys.net/16/914/1998/.

Mager, P. N. and Klimushkin, D. Yu.: Theory of azimuthally smallscale Alfvén waves in an axisymmetric magnetosphere with small but finite plasma pressure, J. Geophys. Res., 107(A11), 1356, doi:1029/2001JA009137, 2002.

Mager, P. N. and Klimushkin, D. Yu.: Spatial localization and azimuthal wave numbers of Alfvén waves generated by driftbounce resonance in the magnetosphere, Ann. Geophys., 23, 3775-3784, 2005,

http://www.ann-geophys.net/23/3775/2005/.

Mann, I. R. and Wright, A. N.: Finite lifetimes of ideal poloidal Alfvén waves, J. Geophys. Res., 100, 23 677-23 686, 1995.

Ozeke, L. G. and Mann, I. R.: Modeling of the properties of high- $m$ Alfvén waves driven by the drift-bounce resonance mechanism, J. Geophys. Res., 106, 15 583-15 597, 2001.
Takahashi, K. and Anderson, B. J.: Distribution of ULF-energy $(f<80 \mathrm{mHz})$ in the inner magnetosphere: a statistical analysis of AMPTE CCE magnetic field data, J. Geophys. Res., 97, 10 751$10769,1992$.

Tataronis J. A. and Grossman, W.: Decay of MHD waves by phase mixing, 1, The sheet-pinch in a plane geometry, Z. Phys., 261, 203-216, 1973.

Vetoulis, G. and Chen, L.: Kinetic theory of geomagnetic pulsations, 3, Global analysis of drift Alfvén-ballooning modes, J. Geophys. Res., 101, 15 441-15 456, 1996.

Yeoman, T. K., Wright, D. M., Chapman, P. J., and Stockton-Chalk, A. B.: High-latitude observations of ULF waves with large azimuthal wavenumbers, J. Geophys. Res., 105, 5453-5462, 2000. 